\section{The cause of intestinal bleeding cannot be missed: the case of primary aortoenteric fistula}

\author{
Bahjat Barakat, ${ }^{1}$ Lucia Calculli, ${ }^{2}$ \\ Raffaele Pezzilli ${ }^{3}$
}

'Department of Emergency; ${ }^{2}$ Department of Diagnostic Medicine and Prevention; ${ }^{3}$ Department of Digestive System, Sant'Orsola-Malpighi Hospital, University of Bologna, Bologna, Italy

\section{Abstract}

Primary aortoenteric fistula (PAEF) is a rare condition of intestinal hemorrhage. We herein report a case of this condition initially suspected by ultrasonographic evaluation and subsequently confirmed by computed tomography. PAEF should be considered a possible cause in patients with gastrointestinal bleeding of uncertain etiology and especially in patients with known aortic aneurysms. The rapid recognition of this condition may save the life of these patients because in untreated subjects the mortality is $100 \%$, frequently due to hypovolemic shock.

\section{Introduction}

Gastrointestinal hemorrhage represents a challenge in Emergency Room. Upper gastrointestinal bleeding is a common medical emergency ranging from 50-150 cases per 100,000 inhabitants. ${ }^{1}$ Most of these bleeding are nonvaricose (esophagitis, Mallory-Weiss syndrome, peptic ulcer, gastric neoplasia, angiodysplasia); primary aorto-enteric fistula is a rare entity but has a high mortality rate and requires early diagnosis for the proper treatment. The accurate clinical history may select those patients having hemorrhage associated to other pathological conditions such as variceal bleeding whereas sometimes the hemorrhage may be due to rare cause. We report a case of massive intestinal bleeding due to a rare condition such as primary aortoenteric fistulas (PAEF).

\section{Case Report}

A 91-year-old man was admitted to the Emergency Room for intestinal bleeding, tachycardia and hypotension (high cardiac rate 120 b.p.m. and arterial blood pressure $100 / 60 \mathrm{mmHg}$ ). His past medical history was unremarkable except for an operation for a pharynx tumor and for the presence of benign prostate hypertrophy. Physical examination revealed a pulsatile mass of the abdomen and the presence of massive rectal bleeding. Patient underwent to fluid resuscitation and blood analyses revealed normal hemoglobin. An ultrasonography of abdomen revealed an abdominal aortic aneurysm of about $10 \mathrm{~cm}$ of diameter partially thrombosed (Figure 1A and B); a dilation of small bowel loops, and the presence of fluid-fluid levels (Figure 2). Echocolor-Doppler confirmed the aneurysm with thrombosis (Figure 1C and D). One hour after admission, the patient general condition started to deteriorate and he became hemodinamically unstable, the hemoglobin dropped to 8 $\mathrm{mg} / \mathrm{dL}$ and 4 units of blood transfusion was administered. After patient stabilization a contrast enhanced-CT scan was carried out. This examination confirmed the presence of a subrenal aortic aneurysm measuring $10 \times 9 \mathrm{~cm}$ with a filiform aortic-enteric fistulous tract and leakage of contrast material in the stomach and ileum (Figures 2 and 3 ).

The patient was immediately transferred to the Emergency Surgical Department where the patient was operated. A prosthetic Goretex tube graft was inserted in the aorta and the fistula, and the intestine was repaired by direct suturing.

The patient had an uneventful recovery and was discharged home after seven days in stable condition.

\section{Discussion}

Primary aortoenteric fistula (PAEF) should be considered a possible cause of gastro-intestinal bleeding of uncertain etiology and especially in patients with known abdominal aortic aneurysm. ${ }^{2}$

PAEF is a rare entity and the reported incidence of PAEF is 0.007 per million ${ }^{3}$ and it is associated with a high mortality. Its occurrence is usually due to erosion of an abdominal aortic aneurysm into the intestinal tract. The most common sites for the fistula are the third and fourth portions of duodenum ${ }^{2}$ and it has also been reported the presence of fistula to the esophagus, stomach, small bowel, colon. ${ }^{1}$

A classical triad of this condition, i.e. gastrointestinal hemorrhage, abdominal mass and abdominal or back pain is uncommon. ${ }^{4-7}$ As in our case, the typical bleeding pattern associated with PAEF is usually intermittent, starting
Correspondence: Raffaele Pezzilli, Department of Digestive System, Sant'Orsola-Malpighi Hospital, Bologna, Italy.

Tel: +39.051.6364148 - Fax: +39.051 .6364148$

E-mail: raffaele.pezzilli@aosp.bo.it

Key words: Primary aortic-enteric fistula; Intestinal bleeding; Ultrasonography; ColorDoppler; Computed tomography.

Conflict of interest: the authors decalre no potential conflict of interest.

Received for publication: 25 February 2016 Revision received: 26 May 2016.

Accepted for publication: 26 May 2016.

This work is licensed under a Creative Commons Attribution 4.0 License (by-nc 4.0).

(c) Copyright B. Barakat et al., 2016

Licensee PAGEPress, Italy

Emergency Care Journal 2016; 12:5845

doi:10.4081/ecj.2016.5845

with a brief herald bleeding followed by major gastrointestinal hemorrhage having often a fatal outcome. ${ }^{8}$ Even if the preoperative examinations are often not helpful and can lead to delayed diagnosis and surgery, we suspected the presence of PAEF by a simple imaging technique such as transabdominal ultrasonography associated with echo-color-Doppler and the final diagnosis was made by contrastenhanced computed tomography that showed the presence of abdominal aneurysm, the linkage between the aneurysm and the duodenum and the presence of blood in the intestinal lumen. In our patient, as usually happens in these subjects, there were risk factors for the presence of atherosclerosis and an upper gastrointestinal bleeding in the absence of an evident source. Definitive treatment includes primary duodenal repair and insertion surgically or radiologically of an aortic stent ${ }^{9-11}$ or aortic aneurismal resection. ${ }^{12}$

\section{Conclusions}

PAEF should be considered a possible cause in patients with gastrointestinal bleeding of uncertain etiology and especially in patients with known aortic aneurysms. The rapid recognition of this condition, that should be considered a true abdominal vascular catastrophe, ${ }^{13}$ saves the life of these patients because in untreated subjects the mortality is $100 \%$, frequently due hypovolemic shock. ${ }^{11}$ 

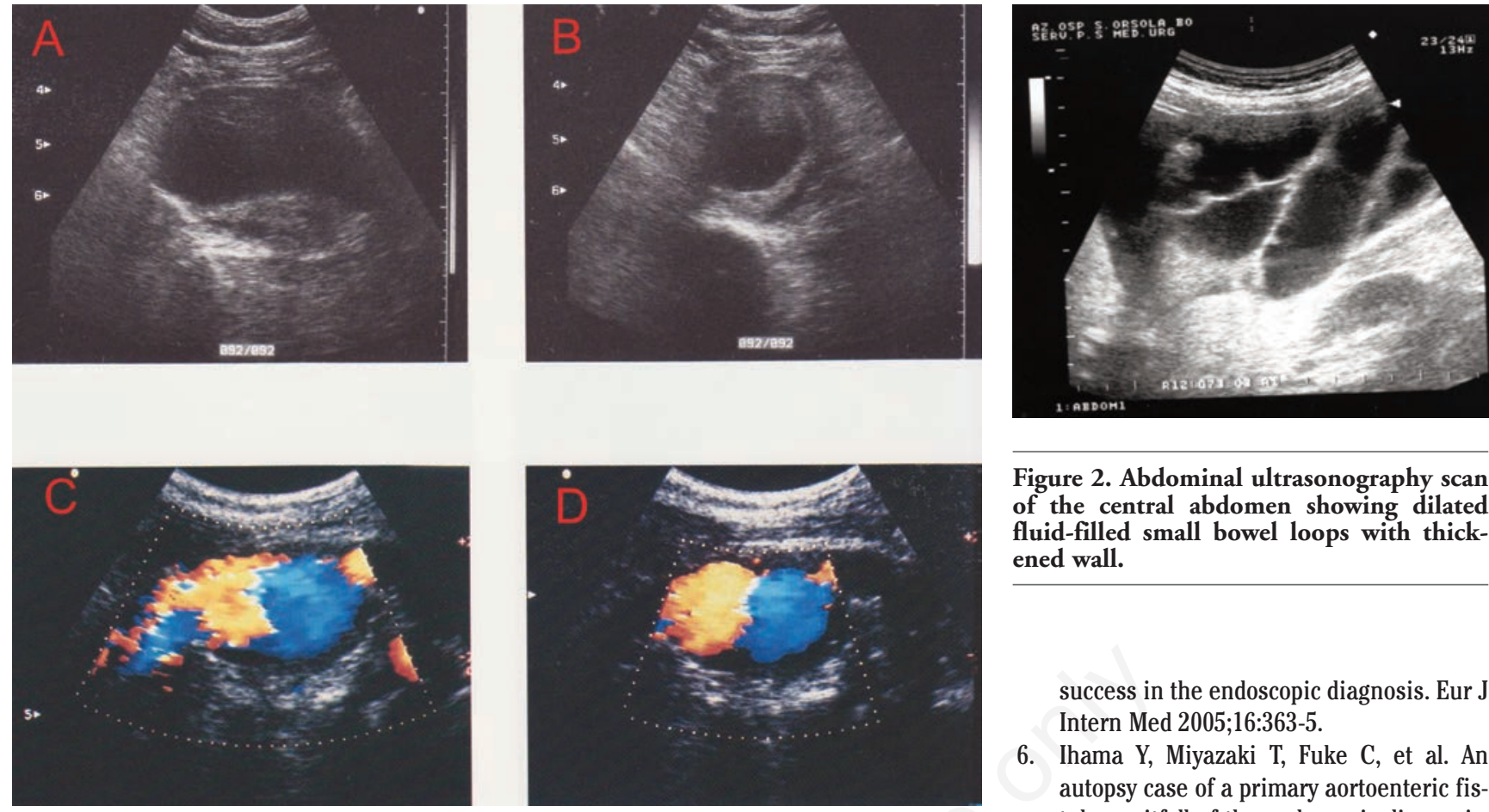

Figure 2. Abdominal ultrasonography scan of the central abdomen showing dilated fluid-filled small bowel loops with thickened wall.

Figure 1. Typical ultrasonographic appearance of abdominal aortic aneurysm: A) longitudinal scan; B) axial scan with clear evidence of the parietal thrombosis delimiting the true lumen; C) and D) echo-color-Doppler confirming the aneurysm with thrombosis.

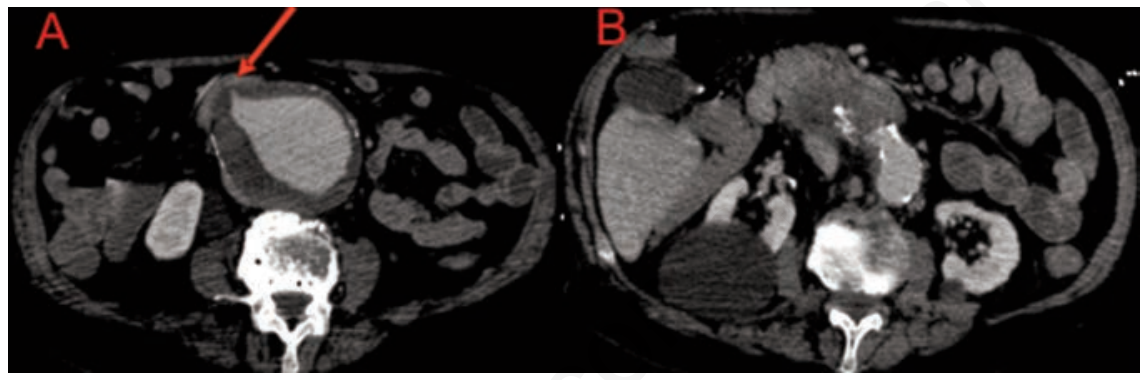

Figure 3. A) Abdominal contrast enhanced computer tomography showing aortic aneurysm of the abdominal aorta with a penetrating right anterolateral ulcer (red arrow); B) the aneurysm causes compressive effect and disappearance of a fat cleavage plane of the duodenum; the duodenum is hyperdense in its inferior-external wall in relation to the hemorrhage.

\section{References}

1. Romera Barba E, Sánchez Pérez A, Bertelli Puche J, et al. Primary aorto-duodenal fistula: a rare but potentially fatal cause of gastrointestinal bleeding. Cir Espan 2015;93:121-3.

2. Alzobydi AH, Guraya SS. Primary aortoduo- denal fistula: a case report. World J Gastroenterol 2013;19:415-7.

3. Parikh K, Ali MA, Wong RC. Unusual causes of upper gastrointestinal bleeding. Gastrointest Endosc 2015;25:583-605.

4. Saers SJ, Scheltinga MR. Primary aortoenteric fistula. Brit J Surg 2005;92:143-52.

5. Delgado J, Jotkowitz AB, Delgado B, et al. Primary aortoduodenal fistula: pitfalls and success in the endoscopic diagnosis. Eur J Intern Med 2005;16:363-5.

6. Ihama Y, Miyazaki T, Fuke C, et al. An autopsy case of a primary aortoenteric fistula: a pitfall of the endoscopic diagnosis. World J Gastroenterol 2008;14:4701-4.

7. Lee CW, Chung SW, Song S, et al. Double primary aortoenteric fistulae: a case report of two simultaneous primary aortoenteric fistulae in one patient. Korean $\mathrm{J}$ Thorac Cardiovasc Surg 2012;45:330-3.

8. Nohr M, Juul-Jensen KE, Balslev IB, Jelnes R. Primary aorto-enteric fistula: a practicable curable condition? Pathogenetic and clinical aspects. Int Angiol 1990;9:278-81.

9. Cendan JC, Thomas JB 4th, Seeger JM. Twenty-one cases of aortoenteric fistula: lessons for the general surgeon. Am Surg 2004;70:583-7.

10. Shapiro M, Addis MD, Ellozy SH, et al. Successful endovascular treatment of bleeding aortoenteric fistula: a case report. Ann Vasc Surg 2006;20:817-9.

11. Khang NC, Zainal AA. Endovascular stent graft repair of aorto-iliac pseudoaneurysms - Hospital Kuala Lumpur experience. Med J Malaysia 2016;71:17-22.

12. Rodrigues dos Santos C, Casaca R, Mendes de Almeida JC, Mendes-Pedro L. Enteric repair in aortoduodenal fistulas: a forgotten but often lethal player. Ann Vasc Surg 2014;28:756-62.

13. Singh M, Koyfman A, Martinez JP. Abdominal vascular catastrophes. Emerg Med Clin N Am 2016;34:327-39. 